\title{
The Role of Surface Plasmons in The Casimir Effect
}

\author{
F. Intravaia, ${ }^{1}$ C. Henkel, ${ }^{1}$ and A. Lambrecht ${ }^{2}$ \\ ${ }^{1}$ Institut für Physik, Universität Potsdam, Am Neuen Palais 10, 14469 Potsdam, Germany \\ ${ }^{2}$ Laboratoire Kastler-Brossel ENS, UPMC, CNRS, Case 74, Campus Jussieu, 75252 Paris Cedex 05, France
}

\begin{abstract}
In this paper we study the role of surface plasmon modes in the Casimir effect. The Casimir energy can be written as a sum over the modes of a real cavity and one may identify two sorts of modes, two evanescent surface plasmon modes and propagative modes. As one of the surface plasmon modes becomes propagative for some choice of parameters we adopt an adiabatic mode definition where we follow this mode into the propagative sector and count it together with the surface plasmon contribution, calling this contribution "plasmonic". We evaluate analytically the contribution of the plasmonic modes to the Casimir energy. Surprisingly we find that this becomes repulsive for intermediate and large mirror separations. The contribution of surface plasmons to the Casimir energy plays a fundamental role not only at short but also at large distances. This suggests possibilities to taylor the Casimir force via a manipulation of the surface plasmons properties.
\end{abstract}

PACS numbers: 42.50.Pq Cavity quantum electrodynamics - 73.20.Mf Collective excitations

\section{INTRODUCTION}

The Casimir force is the archetypal mechanical consequence of vacuum fluctuations in the quantized electromagnetic field. In its simplest form, it gives rise to the attraction of two planar mirrors placed in empty space at zero temperature [1]. The corresponding interaction energy $E$ takes a universal form for perfect reflectors,

$$
E=E_{\text {Cas }}=-\frac{\hbar c A}{4 \pi \aleph L^{3}},
$$

where $L$ is the distance between the mirrors, $A$ their area, and $\hbar$ and $c$ the reduced Planck constant and the speed of light. We abbreviate $\aleph=180 / \pi^{3} \approx 5.8052762$. As usual in thermodynamics, a negative energy corresponds to a binding energy.

The Casimir force was soon observed in different experiments which confirmed its existence [2, 3, 4]. In recent years, technological improvement allowed to reach a precision in the percent range, which makes an accurate comparison to theoretical predictions possible and has prompted a series of refined calculations [5, 6]. Casimir's 1948 derivation of Eq. (1) is based on summing the zero-point energies $\frac{1}{2} \hbar \omega$ of the cavity eigenmodes, taking the difference for finite and infinite separation, and removing the divergences by inserting a high-energy cutoff. He considered an ideal setting with perfectly reflecting mirrors in vacuum. Experiments are however performed with real reflectors, typically metallic mirrors which are good reflectors only at frequencies below the plasma frequency $\left(\omega_{\mathrm{p}} / 2 \pi\right)$ or alternatively at wavelengths much larger than $\lambda_{\mathrm{p}}=2 \pi c / \omega_{\mathrm{p}}$. It has been known since a long time that this has a significant effect on the force, in particular at mirror distances of the order of $\lambda_{p}$ or smaller [7. 8, 9], and precise investigations have been developed recently [5, 10, 11, 12, 13, 14, 15, 16, 17, 18].

A system made from real material mirrors sustains electromagnetic modes which strongly differ with respect to the ideal case, in particular plasma oscillations and surface plasmons (sometimes called surface plasmon polaritons). These are collective electron density waves with energies $\hbar \omega_{\mathrm{p}}$ around ten electron volts (at typical metallic densities). These waves can be quantized and since $\hbar \omega_{\mathrm{p}}$ is larger than any experimentally relevant thermal energy, one can safely consider that bulk plasma modes are in the ground state [19]. This is not quite true for the surface plasmon modes that are confined to the surface of a metallic mirror. Their electronic excitation is accompanied by an electromagnetic field mode that is evanescent inside the cavity [20]. Surface plasmons play an important role in many fields of physics. Let us only mention the plasmon-assisted light transmission through metallic structures [21, 22, 23], or dispersion forces between electronic Wigner crystals that are relevant for biomolecular physics [24]. More generally, evanescent electromagnetic waves have a strong impact on the Casimir-Polder interaction between an atom and a surface as well as on the interaction between two surfaces at differences temperatures [25, 26].

It is well known, indeed, that the Casimir effect, at short distances, is dominated by the coupling between the surface plasmons that propagate on two metallic mirrors. This has been pointed out in 1968 by Van Kampen and co-workers [27] who computed the Casimir energy for $L \ll \lambda_{\mathrm{p}}$ in terms of quasi-electrostatic (or non-retarded) field modes. In this limit the Casimir energy becomes [7, 12, 28]

$$
E \approx \alpha \frac{L}{\lambda_{\mathrm{p}}} E_{\text {Cas }} \quad \text { with } \alpha \approx 1.790 .
$$

which is smaller than Eq.(1). Observe the different power law and the non-universal behavior as the result depends on the material parameter $\lambda_{\mathrm{p}}$. For metals used in modern experiments, $\lambda_{\mathrm{p}}$ lies in the sub-micron range $(107 \mathrm{~nm}$ for $\mathrm{Al}$ and $137 \mathrm{~nm}$ for $\mathrm{Cu}$ and $\mathrm{Au}$ ). This short-distance regime has been studied in much detail since Van Kampen's paper, investigating, for example, materials with a nonlocal response [29, 30].

As the mirror separation increases, retardation has to be taken into account, and Van Kampen's result calls for a generalization. This has been done by Schram in 1973 [31], improving on a previous paper by Gerlach [32]. Schram considered mirrors described by a non-dissipative dielectric 
function, found the electromagnetic modes vibrating between these mirrors, and got the Casimir energy by summing their zero point energies. Among these modes, we find the retarded version of van Kampen's surface plasmon modes. Schram did not analyze separately their contribution and focused on the total energy, using a calculation based on the argument principle. Summerside and Mahanty investigated the joint effect of retardation and nonlocality on the surface plasmon modes at short distances [30].

In this paper we investigate more closely the influence of surface plasmon modes on the Casimir energy, covering both the non-retarded and retarded domains. This permits to explore the experimentally relevant distance range around one micron where current precision experiments are performed. The plasmon modes are identified in a natural way in the sum over electromagnetic modes of the real cavity. We have shown previously that they have peculiar properties [33]: one of them is purely evanescent while the dispersion relation of the other one changes its character from evanescent to propagating inside the cavity (it crosses the light cone). In addition, the combined plasmonic contribution to the Casimir energy has the peculiarity to change sign as a function of distance $L$. Here, we derive and expand on these results in more detail and exhibit closed-form expressions valid at all distances. The main idea is to perform a re-parametrization of the dispersion relations that permit to evaluate analytically the relevant inte- grals. We recover van Kampen's result at short distances and discuss explicitly the asymptotic behaviour in the long distance domain where retardation plays an important role. This regime was not covered in a previous paper by one of us [34] that performs an analysis of surface plasmons in the shortdistance (non-retarded) regime. The analysis of the "photonic modes" (corresponding to waves that propagate in the cavity) will be the object of a following paper. For simplicity, we restrict here to zero temperature, the generalization to finite temperature being straightforward.

The material is organized as follows. The basic method and the cavity modes are introduced in Sec. II. The dispersion relation of the plasmonic modes is analyzed in Sec. III and Appendix A and their contribution to the Casimir energy given in Sec. III A. The Secs. III B and C discuss the short and large distance regimes. Our analysis concludes with a discussion of the sign of the Casimir interaction (Sec. III D) and of alternative splittings of the plasmonic dispersion relations that appeared recently in the literature (Sec. III E).

\section{CASIMIR INTERACTION AND REAL CAVITY MODES}

In 1973 Schram proved the following mathematical identity [31], exploiting the argument principle [5]

$$
E=\sum_{\mu, \mathbf{k}}\left[\sum_{n} \frac{\hbar}{2} \omega_{n}^{\mu}(\mathbf{k})\right]_{L \rightarrow \infty}^{L}=\operatorname{Im} \sum_{\mu, \mathbf{k}} \int_{0}^{\infty} \frac{\mathrm{d} \omega}{2 \pi} \hbar \ln \left(1-r_{\mathbf{k}}^{\mu}[\omega]^{2} e^{2 i k_{z} L}\right)
$$

The left-hand side has the same structure as Casimir's sum over zero point energies, but in this case the relevant modes are those of the real cavity. The notation $[\cdots]_{L \rightarrow \infty}^{L}$ signifies the difference of the expression in brackets for finite and infinite mirror distance $L$. The right-hand side is nothing but the Lifshitz formula for the Casimir energy. Let us recall that Lifshitz adopted in 1955 [7] a fairly different viewpoint and computed the force as the average of the Maxwell stress tensor inside the cavity. He considered the electromagnetic fields as being radiated by fluctuating sources in the medium composing the mirrors, similar to London's derivation of the Van der Waals force between atoms and molecules. The main point of Ref.[31] was to show that the Lifshitz approach yields the same result as the Casimir sum over zero-point energies, provided the mirrors are non-dissipative. This is the case we focus on here.

The modes in Eq. (3) are labelled by their polarization $\mu=$ TE, TM and the wavevector $\mathbf{k} \equiv\left(k_{x}, k_{y}\right)$ parallel to the mirrors; the perpendicular wavevector $k_{z}$ is defined in Eq. (6) below. The $r_{\mathbf{k}}^{\mu}$ are the reflection amplitudes that we take the same for both mirrors. The mode frequencies $\omega_{n}^{\mu}(\mathbf{k})$ are related to the zeros and the branch cuts of [31]

$$
D_{\mu}[\omega ; \mathbf{k}]=1-r_{\mathbf{k}}^{\mu}[\omega]^{2} e^{2 i k_{z} L} .
$$

We adopt here the Fresnel formulas for the reflection amplitudes that for the case of thick mirrors read [36]

$$
r^{T E}=\frac{\kappa-\kappa_{\mathrm{m}}}{\kappa+\kappa_{\mathrm{m}}}, \quad r^{T M}=\frac{\kappa_{\mathrm{m}}-\epsilon[\omega] \kappa}{\kappa_{\mathrm{m}}+\epsilon[\omega] \kappa}
$$

where

$$
\begin{gathered}
k_{z}=\imath \kappa=\imath \sqrt{|\mathbf{k}|^{2}-\omega^{2} / c^{2}} \\
\kappa_{\mathrm{m}}=\sqrt{|\mathbf{k}|^{2}-\epsilon[\omega] \omega^{2} / c^{2}}=\sqrt{\kappa^{2}+\omega_{\mathrm{p}}^{2} / c^{2}}
\end{gathered}
$$

We choose signs for the square roots such that $\operatorname{Re}\left[\kappa_{i}\right]>0$ and $\operatorname{Im}\left[\kappa_{i}\right]<0$ in $\operatorname{Im}[\omega]>0$. This analytical continuation entails that Eq. (4) has no solutions in the upper half plane. 
Finally, $\epsilon[\omega]$ is the dielectric function; in the case of a metal the simplest description is given by the plasma model

$$
\epsilon[\omega]=1-\frac{\omega_{\mathrm{p}}^{2}}{\omega^{2}}
$$

where $\omega_{\mathrm{p}}$ is the plasma frequency, a constant which can be related to the specific physical properties of the metal. Up to $\omega \sim \omega_{\mathrm{p}}$ the dielectric constant differs from unity so that the metal behaves different than the surrounding vacuum. For $\omega \gg \omega_{\mathrm{p}}$ the dielectric constant approaches unity and the metal becomes transparent. This is the way the plasma model implements the high-frequency cutoff for the mirror reflectivity.

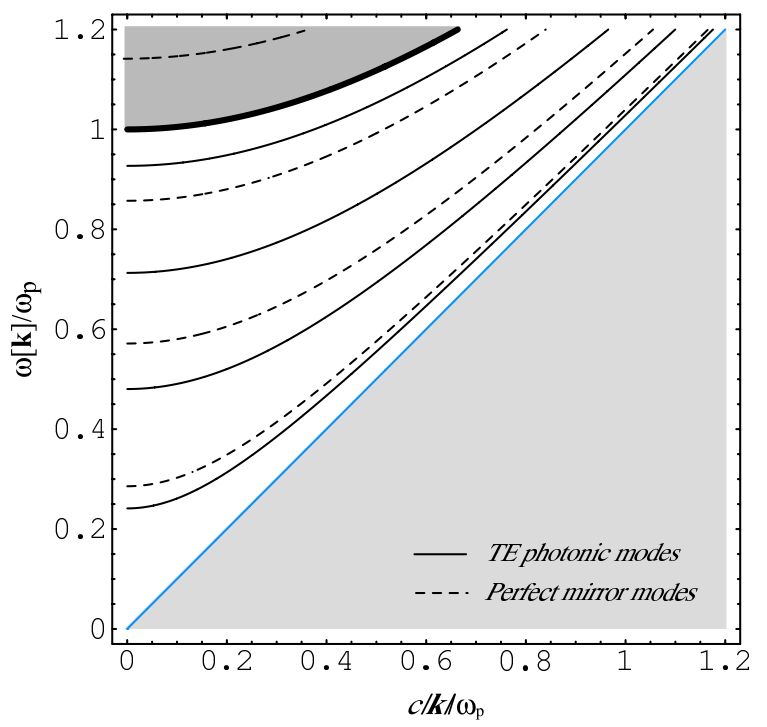

FIG. 1: Dispersion relations for TE-polarized modes between two metallic mirrors described by the plasma model (solid line), compared perfect conductors (dashed line). Mode frequency $\omega(\mathbf{k})$ and wavevector in the mirror plane, $|\mathbf{k}|$, are normalized to the plasma frequency $\omega_{\mathrm{p}}$. Mirror distance $L=1.75 \lambda_{\mathrm{p}}$. The (blue) diagonal line is the light cone below which the field is evanescent in the cavity (evanescent modes). Above the thick solid line, the field propagates through in mirror material (bulk modes).

In this model we neglect all the dissipation phenomena and we impose a local response to the electromagnetic field [36]. From a physical point of view, it is a poor approximation to real metals at low frequencies (dissipation and non-locality, i.e., the anomalous skin effect are predominant) and high frequencies (absorption from intraband transitions). But at any rate, its mathematical simplicity allows explicit calculations to be pushed very far and to understand important physical behaviors. We are going to see that our principal result correspond to a frequency range high enough for the plasma model to be a good description of the metal. Let us stress, however, that the choice of the plasma model is the strongest approximation we make and, within this model, all results we discuss are exact.

The introduction of the dielectric properties of the mirrors leads to a series of important modifications for the field

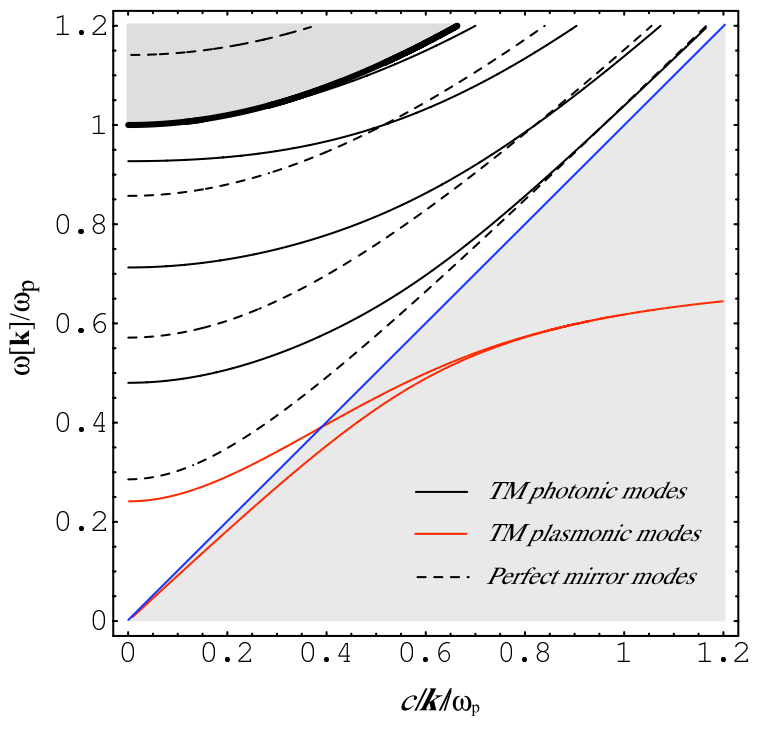

FIG. 2: Same as Fig 1 for TM-polarized modes. The solid red curves represent the plasmonic modes and the black curves the photonic modes. Note that one of the plasmonic modes crosses the light cone.

modes. First of all, even in the simplest case, the plasma model, the dispersion relations $\omega_{n}^{\mu}(\mathbf{k})$ cannot be written in terms of elementary functions. The results of a numerical calculation are shown in figs. 1 and 2 (see details below). As we can see, imperfect reflection modifies the dispersion relation (solid lines) compared to a perfect reflector (dashed lines).

We can distinguish three regions starting from above:

Bulk modes occur for $\omega>\omega_{B}(\mathbf{k})=\left(\omega_{\mathrm{p}}^{2}+c^{2}|\mathbf{k}|^{2}\right)^{1 / 2}$ (shaded above the thick line); they propagate both in the cavity and inside the mirrors. These modes form a continuum that is mathematically represented by a branch cut of Eq. (4) in the complex $\omega$-plane. This has to be taken into account carefully when applying the argument theorem [37]. The associated difficulties have led Schram to work instead with a mirror of finite thickness $d$ where the continuum discretizes [31]. For simplicity, we take here the limit of thick mirrors.

Propagating (ordinary) cavity modes: they occur in the region above the light cone and below the bulk continuum, $c|\mathbf{k}|<\omega<\omega_{B}(\mathbf{k})$. These modes are guided between the mirrors (note that the latter behave like a medium optically thinner than vacuum, $0<\epsilon[\omega]<1$ ), leading to a discrete set of mode frequencies for a given $\mathbf{k}$. In this region, the reflection coefficients (5) have unit modulus and a frequency-dependent phase. This leads to a shift of the cavity modes relative to perfectly reflecting mirrors, as is visible in Figs 12 .

Evanescent modes lie below the light cone, $\omega<c|\mathbf{k}|$ (shaded below the diagonal), and are the main focus of this paper. Their electromagnetic field exponentially decreases when going away from the vacuum-mirror interface, while it is allowed to propagate along the interface. Evanescent fields are of great interest in near field optics because they provide the link to sub-wavelength topographic features of a surface. In the context of the Casimir interaction, they are often underes- 
timated due to their damped nature. We show here, however, that their contribution is all but a small correction, even at large distances [33]. From a mathematical point of view, the optical properties of evanescent modes (reflection and transmission amplitudes) can be obtained from ordinary modes by a well-defined analytical continuation procedure [35]. Solving the dispersion equation (4) in the evanescent sector, one finds two nondegenerate mode frequencies in only one polarization, at least for non-magnetic media. These modes are called "surface plasmons" (or "surface plasmon polaritons") [20, 38, 39]. Their field amplitude decays exponentially away from the interface, and is associated with oscillating surface charge and surface current densities, as required by the equation of con- tinuity (see Fig 3). On an isolated interface, surface plasmons correspond to the pole of $r_{\mathbf{k}}^{T M}[\omega]$; they occur when $\varepsilon[\omega]<-1$ (i.e., $\omega<\omega_{\mathrm{p}} / \sqrt{2}$ ). For two interfaces, two surface plasmons exist and are coupled via their evanescent tails in the cavity. The resulting modes are given by the zeros of Eq. (4) for real $\kappa$ and $\kappa_{\mathrm{m}}$, and will be analyzed in detail in the following.

Summarizing, we can see that for the TE-polarization, all modes lie above the light cone, while for TM-polarization, two modes enter the evanescent region in at least some range of wavevectors. We refer to these modes as "plasmonic"; they are the retarded generalization of van Kampen's coupled surface plasmon modes. Finally, we can re-write the Casimir energy as

$$
E=\underbrace{\sum_{\mathbf{k}}\left[\frac{\hbar \omega_{+}}{2}+\frac{\hbar \omega_{-}}{2}\right]_{L \rightarrow \infty}^{L}}_{\text {plasmonic modes }\left(E_{\mathrm{pl}}\right)}+\underbrace{\sum_{\mu, \mathbf{k}}\left[\sum_{\omega<\omega_{B}} \frac{\hbar \omega_{n}^{\mu}}{2}\right]_{L \rightarrow \infty}^{L}}_{\text {photonic modes }\left(E_{\mathrm{ph}}\right)}+\overbrace{\lim _{d \rightarrow \infty} \sum_{\mu, \mathbf{k}}\left[\sum_{\omega \geq \omega_{B}} \frac{\hbar \omega_{n}^{\mu}}{2}\right]_{L \rightarrow \infty, d}^{L, d}}^{\text {cavity modes }}
$$

These contributions have no physical meaning on their own, i.e. one cannot measure them separately. The only observable is the total Casimir energy, which is the sum of all terms. However, evaluating them separately reveals striking features which suggest new possibilities to taylor the strength and the sign of the Casimir force. In the rest of this paper, we are going to focus our attention on the plasmonic contribution $E_{\mathrm{pl}}$ and shall discuss the remaining contributions to the Casimir energy in another paper.

\section{PLASMONIC MODES}

We plot again in Fig 4 the dispersion relation of the two modes in the first sum of Eq. (8). They end up for large $|\mathbf{k}|$ below the light cone, i.e., the associated field is evanescent both in vacuum and in the mirrors. One branch that we call $\omega_{-}(\mathbf{k})$ lies entirely below the light cone. The second one, $\omega_{+}(\mathbf{k})$ moves continuously into the cavity mode sector as $\mathbf{k}$ is decreased. The inset illustrates the smooth change in the spatial mode function. This mixed character justifies the name "plasmonic" that we use for both modes in the following. We discuss in Appendix A some general features of their dispersion relations that can be obtained explicitly despite the fact that we have to deal with implicit functions.

\section{A. Contribution to the Casimir energy}

The plasmonic contribution is defined as the first sum on the r.h.s. of eq. (8), namely

$$
E_{\mathrm{pl}}=\sum_{\mathbf{k}}\left[\frac{\hbar \omega_{+}}{2}+\frac{\hbar \omega_{-}}{2}\right]_{L \rightarrow \infty}^{L}
$$

Both modes tend to $\omega_{0}(K)$ for $L \rightarrow \infty$ so that we subtract the zero-point energy for two isolated surface plasmons. We are thus measuring the interaction energy arising from the coupling between the surface plasmons.

Replacing the k-summation by an integral and using the scaled variables introduced in A1, we get

$$
E_{\mathrm{pl}}=\frac{\hbar c A}{2 L^{3}} \int_{0}^{\infty} \frac{K d K}{2 \pi}\left(\Omega_{+}(K)+\Omega_{-}(K)-2 \Omega_{0}(K)\right) .
$$

To check the convergence at large $K$, we use the parametrization of Eqs. A3 and find the estimate

$$
\Omega_{ \pm}^{2}(K)-\Omega_{0}^{2}(K) \rightarrow \pm \frac{1}{2} \Omega_{\mathrm{p}}^{2} e^{-K}+O\left(e^{-2 K}\right)
$$

provided $K \gg \max \left(1, \Omega_{\mathrm{p}}\right)$. For further details, see the discussion around Eq. (18). The difficulty in Eq. 10 is that the dispersion relations $\Omega_{ \pm}(K)$ are only known implicitly in the general case. We now show that using the parametrization of Appendix A, the integrand can be brought into an explicit and elementary form. 


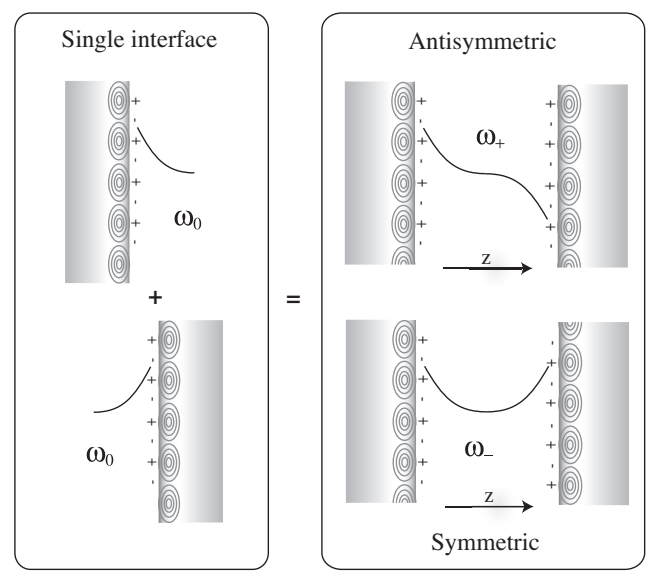

FIG. 3: A surface plasmon mode is associate with an electronic charge oscillation bound to the surface of a body. For a single body the associated electric field is evanescent and, for a plane interface, the plasmon can be excited only by approaching from the vacuum side a medium with higher index of refraction and illuminating the latter in total internal reflection. Approaching two surfaces, the two respective surface plasmons couple through their evanescent field tails. A frequency splitting occurs giving rise to two new modes, the plasmonic modes. The antisymmetric $\left(\omega_{+}\right)$and the symmetric $\left(\omega_{-}\right)$ mode have higher resp. lower energy than the isolated (non-coupled) mode $\omega_{0}$. The Casimir force associated with $\omega_{+}$is then an antibinding force (repulsive) while the $\omega_{-}$modes contribute an attractive force. The plasmonic Casimir force arises from the (distancedependent) balance of the two contributions.

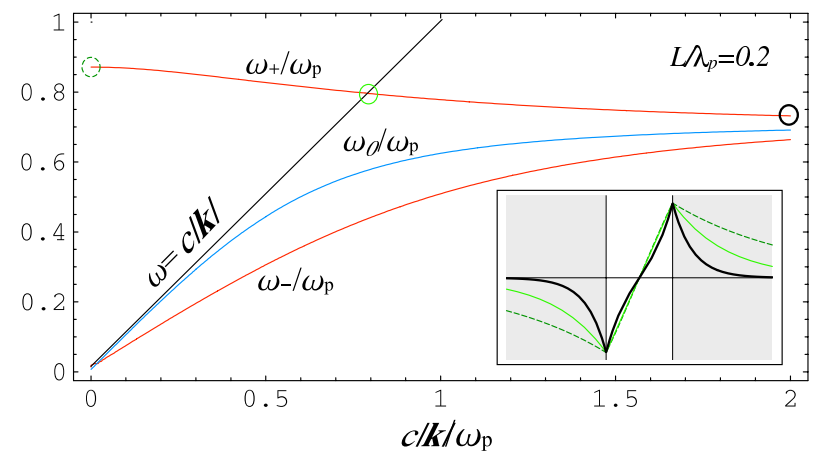

FIG. 4: A plot of the plasmonic dispersion relations $\omega_{+}(\mathbf{k}), \omega_{0}(\mathbf{k})$, $\omega_{-}(\mathbf{k})$, as function of $|\mathbf{k}|$ for $L=0.2 \lambda_{\mathrm{p}}\left(\lambda_{\mathrm{p}}=2 \pi c / \omega_{\mathrm{p}}\right)$. Frequencies and wavevectors are scaled to the plasma frequency $\omega_{\mathrm{p}}$ and $\omega_{\mathrm{p}} / c$, respectively. Inset: magnetic field amplitude for chosen points along the branch $\omega_{+}(\mathbf{k})$, as labelled by the circles.

It is useful to scale the energy Eq. 10 , to the perfect-mirror Casimir energy $E_{\text {Cas }}$ [Eq.(1]]

$$
\begin{gathered}
E_{\mathrm{pl}}=\eta_{\mathrm{pl}} E_{\mathrm{Cas}} \\
\eta_{\mathrm{pl}}=-\aleph \int_{0}^{\infty} \sum_{a= \pm, 0} c_{a} \Omega_{a}(K) K d K
\end{gathered}
$$

with $c_{+}=c_{-}=1, c_{0}=-2$. We call $\eta_{\mathrm{pl}}$ the correction factor for the plasmonic Casimir energy; note that it depends on the distance only via the dimensionless parameter $\Omega_{\mathrm{p}}$.

For each of the branches $\Omega_{a}(K)$, we now change to the integration variable $z=(\kappa L)^{2}$. The Jacobian (the prime denotes the derivative)

$$
d K^{2}=2 K d K=d z+g_{a}^{\prime}(z) d z
$$

with $g_{a}(z)$ defined in Appendix A leads to

$$
\eta_{\mathrm{pl}}=-\frac{\aleph}{2} \sum_{a= \pm, 0} c_{a} \int_{\Gamma_{a}}\left(1+g_{a}^{\prime}(z)\right) \sqrt{g_{a}(z)} d z
$$

The integration paths are now $\Gamma_{+}=-z_{+} \ldots \infty$, and $\Gamma_{-, 0}=$ $0 \ldots \infty$ where $z_{+}$is defined in Eq. A8 .

One of the two terms under the integral can be integrated immediately, leading to

$$
\sum_{a= \pm, 0} c_{a} \int_{\Gamma_{a}} g_{a}^{\prime}(z) \sqrt{g_{a}(z)} d z=\frac{2}{3} \sum_{a= \pm, 0} c_{a}\left[g_{a}^{3 / 2}(z)\right]_{\Gamma_{a}}
$$

where the function in brackets has to be evaluated at the end points of the respective integration domains. The upper limit contributions $(z=\infty)$ cancel under the subtractions. At the lower limit, $g_{-, 0}^{3 / 2}(0)$ vanishes because the dispersion relations reach $\omega=0$ (see Sec A. We are thus left with $g_{+}^{3 / 2}\left(-z_{+}\right)=$ $z_{+}^{3 / 2}$.

Putting the propagating sector of the mode $\Omega_{+}(K)$ into a separate integral, the correction factor for the plasmonic Casimir energy can be rewritten as

$\eta_{\mathrm{pl}}=-\frac{\aleph}{2}\left[\int_{0}^{\infty} \sum_{a} c_{a} \sqrt{g_{a}(z)} d z+\int_{-z_{+}}^{0} \sqrt{g_{+}(z)} d z-\frac{2}{3} z_{+}^{3 / 2}\right]$

In the first integral, the functions $g_{a}(z)$ are real. For $z \rightarrow \infty$, the functions $g_{ \pm}(z)$ approach $g_{0}(z)$ exponentially fast. An expansion in $e^{-\sqrt{z}}$ leads to

$$
\sum_{a} c_{a} \sqrt{g_{a}(z)} \approx-\Omega_{\mathrm{p}} e^{-2 \sqrt{z}} f\left(z / \Omega_{\mathrm{p}}^{2}\right)
$$

where the function $f\left(z / \Omega_{\mathrm{p}}^{2}\right)$ is bounded and tends to $1 /(4 \sqrt{2})$ for $z \gg \Omega_{\mathrm{p}}^{2}$. This secures the convergence at large $z$ of the first integral in Eq.(17). The second integral is finite because $g_{+}(z)$ is bounded and the integration domain is finite [see Eq. A99]. Both the second integral and the third term in Eq.17] are related to the propagating segment of the plasmonic mode $\Omega_{+}(K)$.

The great advantage of Eq.(17) compared to Eq.(10) is that now the integrands are expressed in terms of simple analytic functions and there is no need to integrate implicit functions whose evaluation is only possible numerically. We also gain for analytical calculations since the discussion of the distance dependence (via the parameter $\Omega_{\mathrm{p}} \propto L / \lambda_{\mathrm{p}}$ ) can be done in a transparent way. We show in the following that one gets 
asymptotic expressions for small and large values of $\Omega_{\mathrm{p}}$, the only variable on which the correction factor $\eta_{\mathrm{pl}}$ depends after the integration.

Fig 5 shows a plot of $\eta_{\mathrm{pl}}$ as function of $L / \lambda_{\mathrm{p}}=\Omega_{\mathrm{p}} /(2 \pi)$. Note the increase linear in $L$ for small distances and a sign change at large $L$, with a power law $\propto L^{1 / 2}$. In the next two sections we analyze these limits analytically. At short distance $\eta_{\mathrm{pl}}$ reproduces exactly the correction factor known for the total Casimir energy.

\section{B. Short distance asymptotics}

The distance enters the correction factor $\eta_{\mathrm{pl}}$ [Eq. [17] ] via the dimensionless parameter $\Omega_{\mathrm{p}}$, and we get the short-distance asymptotics in the limit $\Omega_{\mathrm{p}} \ll 1$. This has been discussed in previous papers [28, 32, 34, 40], but the asymptotics turns out to be tricky at next-to-leading order.

The first order expansion in $\Omega_{\mathrm{p}}$ of the functions $g_{a}(z)$ yields [28]

$$
\eta_{\mathrm{pl}} \approx \alpha \frac{\Omega_{\mathrm{p}}}{2 \pi}=\alpha \frac{L}{\lambda_{\mathrm{p}}}
$$

where the numerical constant $\alpha \approx 1.790$ arises from

$$
\alpha=-\frac{\pi \aleph}{\sqrt{2}} \int_{0}^{\infty}\left(\sqrt{1+e^{-\sqrt{z}}}+\sqrt{1-e^{-\sqrt{z}}}-2\right) d z
$$

The separate contributions of the modes $\Omega_{+}(K)$ and $\Omega_{-}(K)$ are $\alpha_{+} \approx-12.225$ (repulsive) and $\alpha_{-} \approx 14.015$ (attractive). The plasmonic Casimir energy in this regime thus scales like $A \hbar \omega_{\mathrm{p}} / L^{2}$ and is reduced compared to the perfect mirror case $\left(\eta_{\mathrm{pl}} \ll 1\right)[41]$.

The contribution of the propagating part of $\omega_{+}$is of the third order in $\Omega_{\mathrm{p}}$ [see Eq.A9]

$$
\int_{-z_{+}}^{0} g_{+}(z) d z \stackrel{\Omega_{\mathrm{p}} \ll 1}{\longrightarrow} g_{+}(0) z_{+} \approx \Omega_{+0} \Omega_{\mathrm{p}}^{2} \approx \Omega_{\mathrm{p}}^{3}
$$

and can therefore be neglected. The same argument holds for the term $\left(z_{+}\right)^{3 / 2} \approx \Omega_{\mathrm{p}}^{3}$. In other words, the plasmonic contribution comes essentially from the evanescent sector $(z>0)$. We note that the result 19a yields exactly the short-distance behavior of the full Casimir energy which is thus dominated at short distance by the interactions between surface plasmons [28, 32, 34, 40].

Note that Eq. 19a follows from an expansion of the $g_{a}(z)$ to first order in $\Omega_{\mathrm{p}}$. It is worth stressing that this expansion scheme does not work at higher orders, the series being an asymptotic one and not uniformly convergent. Each integral obtained by this method at higher orders is divergent, except the first one given in Eq. 19a. To avoid this problem, we use an alternative method and write the functions $g_{ \pm}(z)$ as follows

$$
g_{ \pm}(z)=g_{0}(z) \frac{1 \pm e^{-\sqrt{z}}}{1 \pm \rho}
$$

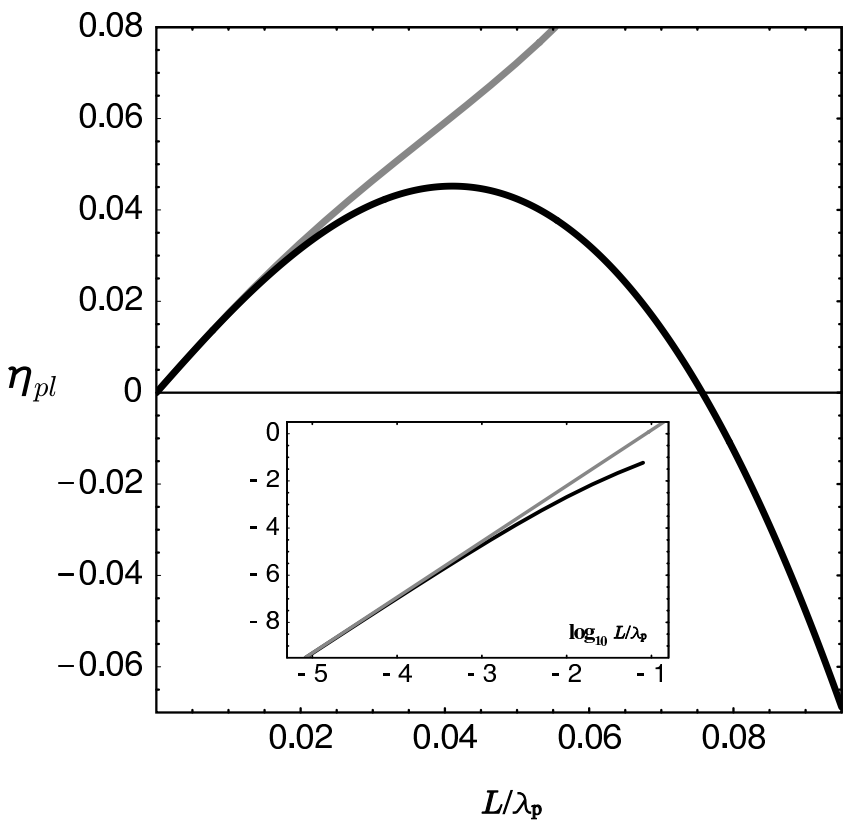

FIG. 5: A plot of the plasmonic Casimir energy, normalized to the perfect mirror case (i.e., the plasmonic correction factor $\eta_{\mathrm{pl}}$ ) vs. the scaled distance $L / \lambda_{\mathrm{p}}$. Light gray solid line: approximation 24. The inset illustrates the short-distance behaviour beyond the linear term: we plot $\left[\eta_{\mathrm{pl}}-\alpha\left(L / \lambda_{\mathrm{p}}\right)\right] / \Omega_{p}^{3}$ (black line) and compare to the expression $a+b \log \left(2 \pi L / \lambda_{\mathrm{p}}\right)$ in Eq.24 (light gray line).

with

$$
\rho=e^{-\sqrt{z}} \frac{g_{0}(z)-\Omega_{\mathrm{p}}^{2} / 2}{\Omega_{\mathrm{p}}^{2} / 2}
$$

Now for $z>0,|\rho|$ is bounded by unity and decays rapidly to zero as $z \rightarrow \infty[28]$. To compute the integral of $\left[g_{ \pm}(z)\right]^{1 / 2}$, we expand in powers of $\rho$ and get the series

$$
\left[g_{ \pm}(z)\right]^{1 / 2}=\sqrt{g_{0}(z)\left(1 \pm e^{-\sqrt{z}}\right)} \sum_{n=0}^{\infty} \frac{\sqrt{\pi}}{\Gamma\left(\frac{1}{2}-n\right)} \frac{( \pm \rho)^{n}}{n !}
$$

where $\Gamma(\cdot)$ is the gamma function. Taking the $n=0$ term, the integration over $z$ leads to $19 \mathrm{a}$. Higher order terms can be calculated explicitly, but the resulting expressions are cumbersome and will not be reported here. Including the next-toleading order terms, we find

$$
\eta_{\mathrm{pl}} \approx \alpha \frac{\Omega_{\mathrm{p}}}{2 \pi}+\left(a+b \log \Omega_{\mathrm{p}}\right) \Omega_{\mathrm{p}}^{3}
$$

where $a \approx 0.63$ and $b=\aleph / 4 \sqrt{2} \approx 1.026$. This is plotted as gray line(s) in Fig 5, the inset providing a zoom on the cubic and logarithmic terms (see caption). Note that the term $a \Omega_{\mathrm{p}}^{3}$ gives a distance-independent correction to the Casimir energy and cancels when the force is computed. The presence of the logarithmic correction is due to the non-uniform convergence of the asymptotic series. We find from (24) that the Casimir force does not feature a logarithmic correction at short distance. 


\section{Large distance asymptotics}

The curve $\eta_{\mathrm{pl}}(L)$ in Fig 5 shows that the plasmonic mode contribution is negative (repulsive) at distances $L \gtrsim 0.08 \lambda_{\mathrm{p}}$. Mathematically, this can easily be seen from the large $\Omega_{\mathrm{p}}$ asymptotics of $\eta_{\mathrm{pl}}$. One can check that the integrand of the first integral in Eq. (17) is significantly different from zero only for $z \sim 1$. This suggests the following expansion of the $g_{a}(z)$ for $\Omega_{\mathrm{p}} \gg 1$

$$
\begin{gathered}
g_{+}(z) \approx \sqrt{\Omega_{\mathrm{p}}} \sqrt{\sqrt{z} \operatorname{coth}\left(\frac{1}{2} \sqrt{z}\right)} \\
g_{-}(z) \approx \sqrt{\Omega_{\mathrm{p}}} \sqrt{\sqrt{z} \tanh \left(\frac{1}{2} \sqrt{z}\right)} \\
g_{0}(z) \approx \sqrt{\Omega_{\mathrm{p}}} \sqrt[4]{z}
\end{gathered}
$$

Moreover, the expansion to leading order in $\Omega_{\mathrm{p}}^{2} \gg|z|$ can also be performed in the integral over the propagating sector in (17) because the integration domain is limited to $-\pi^{2} \approx$ $z_{+} \leq z \leq 0$. Finally, we find that the integrated term in Eq.17) gives a negligible contribution so that to leading order, $\eta_{\mathrm{pl}}=-\Gamma \sqrt{\Omega_{\mathrm{p}}}[40]$ with

$$
\begin{aligned}
\Gamma & =\aleph \int_{0}^{\infty} y^{3 / 2}\left(\sqrt{\operatorname{coth}\left[\frac{y}{2}\right]}+\sqrt{\tanh \left[\frac{y}{2}\right]}-2\right) d y \\
& +\aleph \int_{0}^{\pi} y^{3 / 2} \sqrt{\cot \left[\frac{y}{2}\right]} d y
\end{aligned}
$$

This expression can be evaluated numerically, giving as result $\Gamma=29.75$ (i.e. the sum of 8.90 ( + mode, evanescent sector), -7.23 ( - mode, evanescent sector), and 28.09 ( + mode, propagating sector). Note the large contribution of the propagating segment and the near cancellation of the two evanescent branches.

Since $\eta_{\mathrm{pl}}$ is negative at large distances, the plasmonic contribution provides a repulsive contribution to the Casimir interaction that scales like $+A \hbar \sqrt{\omega_{\mathrm{p}} c} / L^{5 / 2}$. This is balanced in the total Casimir energy by the contributions of photonic modes (cavity and bulk modes), recovering the attractive large-distance power law $E_{\text {Cas }} \propto-A \hbar c / L^{3}$.

\section{Cancellations and signs}

We conclude our analysis by suggesting an interpretation of the signs of the plasmonic contributions to the Casimir energy. It is clear that $E_{\mathrm{pl}}$ is due to the shift in the plasmon mode frequency relative to the isolated interface [see Eq. [10]].

This can be also interpreted as a reshuffling of the density of modes due to the coupling by the interface, the total number of modes remaining constant. To make this more quantitative, we re-write the plasmonic Casimir energy as

$$
\frac{\hbar}{2}\left[\sum_{\mathbf{k}, a= \pm} \omega_{a}(\mathbf{k})\right]_{L \rightarrow \infty}^{L}=\frac{\hbar}{2} \sum_{a= \pm} \int_{0}^{\infty} \mathrm{d} \omega \omega\left[\rho_{a}(\omega)-\rho_{0}(\omega)\right]
$$

where the mode densities are defined as usual by $(a= \pm, 0)$

$$
\rho_{a}(\omega)=\sum_{\mathbf{k}} \delta\left(\omega-\omega_{a}(\mathbf{k})\right),
$$

that depend on the distance $L$ for $a= \pm$. Note that the $\omega$ integral in 27) does not converge if taken over the $\rho_{a}(\omega)$ alone. This is due to the flat large- $k$ asymptote of the plasmonic dispersion relations. More explicitly, the density of modes can be calculated as

$$
\rho_{L, a}(\omega)=\frac{A k_{a}(\omega ; L)}{2 \pi}\left|\frac{\mathrm{d} k_{a}(\omega ; L)}{\mathrm{d} \omega}\right|
$$

where, $k_{a}(\omega)$ is the inverse function to $\omega_{a}(\mathbf{k})$, and the derivative is just the inverse group velocity at a given frequency $\omega$. We find a behaviour $\rho_{a}(\omega) \propto\left(\omega_{\mathrm{sp}}^{2}-\omega^{2}\right)^{-2}$ when $\omega$ approaches the asymptotic value $\omega_{\mathrm{sp}} \equiv \omega_{\mathrm{p}} / \sqrt{2}$ of the dispersion relation (the surface plasmon resonance in the quasistatic limit). This peak is exactly cancelled in the difference $\delta \rho_{ \pm}(\omega) \equiv \rho_{ \pm}(\omega)-\rho_{0}(\omega)$ that we plot in Fig 6 for a given distance $L$. The precise behaviour of the curves changes with the distance (at smaller $L$, for example, $\rho_{+}(\omega)$ is nonzero for $\omega>\omega_{\mathrm{sp}}$ ), but the following qualitative features are stable. (i) The mode $\omega_{+}(k)$ shows a gap between 0 and $\omega_{+}(0)$, and the difference $\delta \rho_{+}(\omega)$ is only due, for $\omega<\omega_{+}(0)$, to the subtracted isolated surface plasmon (dashed line). Just at this frequency, the mode density $\rho_{+}(\omega)$ jumps to a positive value. This behaviour is due to the quadratic shape of the lower band edge in $\omega_{+}(k)$. As $\omega \rightarrow \omega_{\text {sp }}, \delta \rho_{+}(\omega)>0$ because $\omega_{+}(k)$ is shifted upwards relative to $\omega_{0}(k)$ (the group velocity is smaller). This mode is hence an 'anti-binding one' [34]. (ii) The mode $\omega_{-}(k)$ has a linear dispersion for small $k$, and the difference in mode density can be worked out as the positive quantity $\delta \rho_{-}(\omega) \propto \omega / \Omega_{\mathrm{p}} \propto \omega L$ (dashed line). This mode is hence anti-binding in this region as well. (iii) Near the frequency $\omega_{\text {sp }}$, the mode $\omega_{+}(k)\left[\omega_{-}(k)\right]$ gives a repulsive [attractive] contribution to the Casimir energy, respectively. Summing over both modes yields a repulsive or attractive result depending on $L$, because the relative weight of the binding and anti-binding regions changes. Coming back to frequency shifts, it is easy to see from the large $K$ expansion of Eqs. A3 that the following inequality holds

$$
\left|\omega_{-}(\mathbf{k}, L)-\omega_{0}(\mathbf{k})\right|>\left|\omega_{+}(\mathbf{k}, L)-\omega_{0}(\mathbf{k})\right| \quad\left(\mathbf{k} \gg \omega_{\mathrm{p}} / c\right),
$$

At short distance, the plasmonic Casimir energy (which is actually the total Casimir energy) is thus attractive, as is well known (see also Eq 18).

Let us finally note that as one moves away from the large$k$ regime, retardation becomes increasingly important. The change in sign of the plasmonic Casimir energy can thus be seen as well as a consequence of the finite speed of light.

\section{E. Cutting the mode branch}

Recently, there has been some discussion on the way to split the field modes into photon-like and plasmon-like 


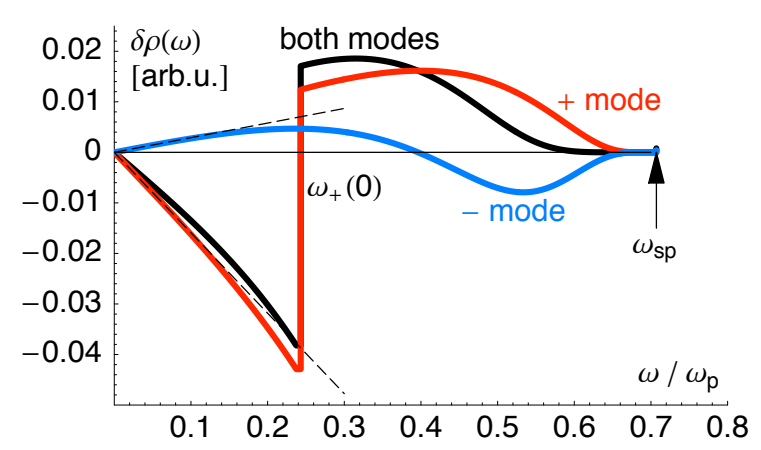

FIG. 6: Change in DOS, $\rho_{L, \pm}(\omega)-\rho_{\infty}(\omega)$, for the two plasmonic modes, as defined in Fig $4=1.75 \lambda_{\mathrm{p}}\left(\Omega_{\mathrm{p}} \approx 10.996\right)$. Dashed lines: small-frequency approximations discussed in the text. The frequency is scaled to $\omega_{\mathrm{p}}$, and the isolated surface plasmon resonance is at $\omega_{\mathrm{sp}}=\omega_{\mathrm{p}} / \sqrt{2}$.

parts [40, 46]. We comment in this section on the numbers one can obtain when the plasmonic mode $\omega_{+}(k)$ is segmented in a different way. (The mode $\omega_{-}(k)$ is subject to no controversy.) The main conclusion we draw from this discussion is that the large distance behavior is dominated by mode branches near the light line. In addition, the sign is sensitive to the chosen subtraction (renormalization), and it may happen that under this procedure, a pure evanescent branch ends up being counted among photonic modes. We also suggest that the branch of the plasmonic mode $\omega_{+}(k)$ that enters the propagating sector is perhaps one of the best examples of Casimir repulsion due to a standing wave mode. Consider the corresponding pressure: it is repulsive due to photons bouncing on the mirrors. The attractive force for a perfect cavity arises, all things told, from the subtraction of a similarly repulsive pressure from a standing wave mode continuum (reflected from the mirrors' backfaces). Now, the counterpart for the plasmonic mode is a single-interface evanescent mode with zero pressure so that the repulsive force survives the subtraction.

Bordag [40] is calling 'plasmon mode' only the evanescent branch of $\omega_{+}(k)$ that exists for $k \equiv|\mathbf{k}|>k_{\mathrm{c}} \equiv$ $\omega_{\mathrm{p}} /\left(c \sqrt{1+\Omega_{\mathrm{p}} / 2}\right.$ ) (see Fig 7 top). The segment within the light cone actually does not appear explicitly in Eq.(24) of Ref.[40], but is implicitly contained in the total Casimir energy (the photonic contribution is computed by subtracting the plasmonic one). The evanescent segment of $\omega_{+}(k)$ is renormalized by subtracting the isolated surface plasmon, $\omega_{0}(k)$, over the same range $k_{\mathrm{c}}<k<\infty$, as shown in Fig77(top). The range $0<k<k_{\mathrm{c}}$ is left out (although it depends on $L$ via $k_{\mathrm{c}}$ ). This subtraction is sufficient to get a vanishing energy as $L \rightarrow \infty$ because $\omega_{+}(k) \rightarrow \omega_{0}(\mathbf{k})$ exponentially fast for $k>k_{\mathrm{c}}$. (In addition, $k_{\mathrm{c}} \rightarrow 0$.) The integration over the branches chosen in Ref.[40] corresponds to the following cor-
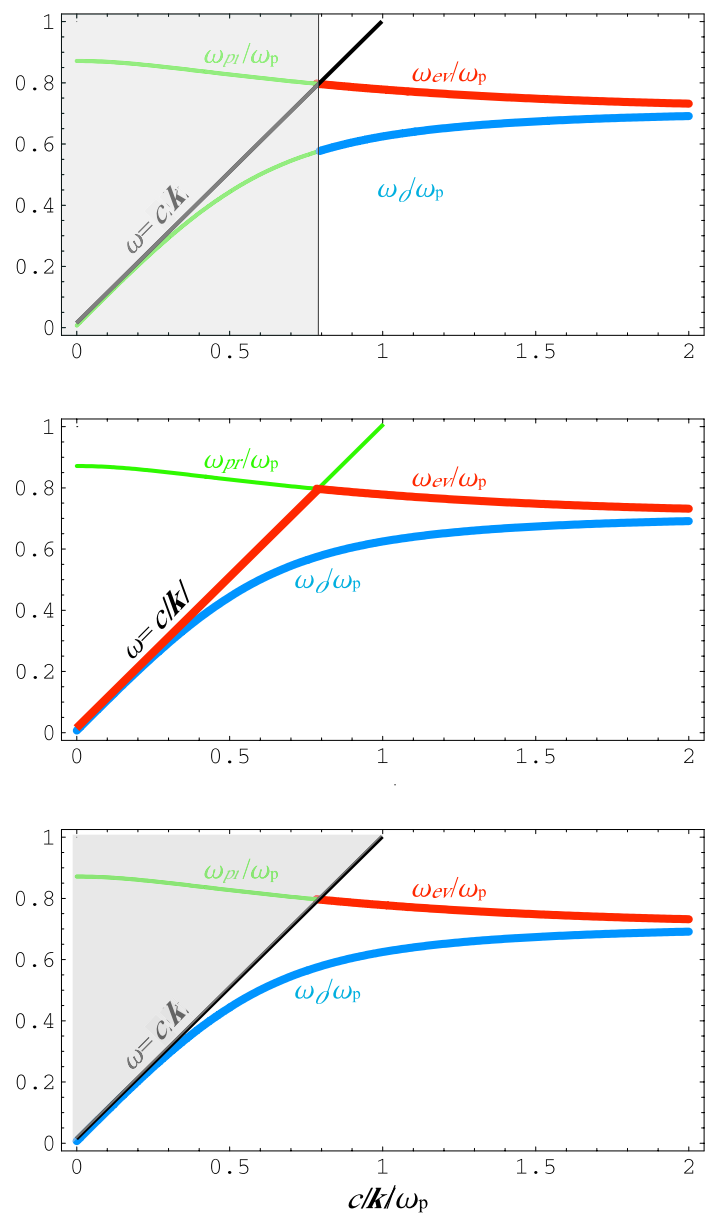

FIG. 7: Illustration of different segmentation of the plasmonic modes and the chosen renormalization. Thick lines mark the segments that are taken into account in the different approaches. We write $\omega_{\mathrm{pr}}$ and $\omega_{\text {ev }}$ for those parts of the mode $\omega_{+}(k)$ where the field between the mirrors is propagating or evanescent, respectively.

Top: Bordag [40], the modes $\omega_{+, 0}(k)$ (red, blue) start at the wavevector $k_{\mathrm{c}}$ where $\omega_{+}(k)$ reaches the light cone. Middle: one possibility suggested by the comment of Lenac [46]. The mode $\omega_{\text {ev }}(k)$ is continued, for $0 \leq k \leq k_{\mathrm{c}}$, by the light line $\omega=c k$ (red) and renormalized by the entire branch of $\omega_{0}(k)$ (blue). A particular splitting of the Lifshitz formula into propagating and evanescent modes turns out to yield the same result. Bottom: another possibility compatible with Lenac's paper. Only the evanescent branch $\omega_{\text {ev }}(k)\left(k \geq k_{\mathrm{c}}\right.$, red) is taken into account and renormalized by $\omega_{0}(k)(k \geq 0$, blue $)$.

rection factor to the Casimir energy:

$$
\begin{aligned}
& \eta_{\mathrm{B}}=\eta_{\mathrm{L}} \\
& -\frac{\aleph}{2}\left[\int_{0}^{z_{\mathrm{c}}} \sqrt{g_{0}(z)} d z+\frac{2}{3}\left(\Omega_{0}^{3}\left(K_{\mathrm{c}}\right)-K_{\mathrm{c}}^{3}\right)\right]
\end{aligned}
$$

with

$$
\eta_{\mathrm{L}}=-\frac{\aleph}{2} \int_{0}^{\infty} \sum_{a= \pm, 0} c_{a} \sqrt{g_{a}(z)} d z
$$


Here, $K_{\mathrm{c}}=k_{\mathrm{c}} L$ and $z_{\mathrm{c}}$ solves the equation $K_{\mathrm{c}}^{2}=g_{0}\left(z_{\mathrm{c}}\right)$ (at this parameter value, the dispersion relation $\omega_{0}(k)$ reaches $k=k_{\mathrm{c}}$ ). We have checked that at short distance, this correction is negligible compared to the leading order $\eta_{\mathrm{pl}} \propto \omega_{\mathrm{p}} L$. At large distance, however, the integrals in $(31$ are both of or$\operatorname{der} \Omega_{\mathrm{p}}^{1 / 2}$ (see Section IIIC) and the difference $\Omega_{0}^{3}\left(K_{\mathrm{c}}\right)-K_{\mathrm{c}}^{3}$, too. Their contributions come with different signs, leading in the end to a correction factor that is attractive and scales like $\eta_{\mathrm{B}} \approx 1.6240 \Omega_{\mathrm{p}}^{1 / 2}$ at large distance.

A similar analysis can be done for the mode definition sketched in Fig 7 (middle): the plasmonic mode is continued along the light line for $k<k_{\mathrm{c}}$ and renormalized by the entire dispersion branch $\omega_{0}(k)$. For $L \rightarrow \infty$, as $k_{\mathrm{c}} \rightarrow 0$, the renormalized energy vanishes. The corresponding correction factor is given by $\eta_{\mathrm{L}}$ [Eq. [32]] which does not contain any integrated term. The short-distance behaviour is the same as in the present paper, and at large distance, we have $\eta_{\mathrm{L}}(L) \approx-1.6600 \Omega_{\mathrm{p}}^{1 / 2}$. This corresponds to repulsion as with our convention, but with a smaller numerical coefficient. We argue below that this result can also be obtained by a splitting of the Lifshitz formula for the Casimir energy. Let us mention that if the segment $0 \leq k \leq k_{\mathrm{c}}$ of the light line is not taken into account (Fig 7 , bottom)) then the large distance behaviour shows an attractive term $\eta \propto \Omega_{\mathrm{p}}^{3 / 2}$. Both results do not fit with the curves presented by Lenac [46], although our calculation tries to follow the spirit of his description. It is not clear to us from his sparse description which renormalization scheme was used in the end.

The Lifshitz approach to the Casimir energy leads to the correction factor $\eta_{\mathrm{L}}$ as follows. We write the right-hand side of Eq. (3) in the equivalent form

$$
E_{\mathrm{L}}=-\operatorname{Im} \sum_{\mu, \mathbf{k}} \int_{0}^{\infty} \frac{\mathrm{d} \omega}{2 \pi} \hbar \omega \frac{d}{d \omega} \log D_{\mu}[\omega, \mathbf{k}]
$$

where the dispersion function $D_{\mu}[\omega, \mathbf{k}]$ is defined in (4). This expression has a structure very similar to the so-called "argument principle" where the zeros (and poles) of the argument of the logarithm define the eigenfrequencies of the system (of the reference system), respectively [5], and each mode contributes its zero point energy. In other words, the imaginary part of the logarithmic derivative can be read as a density of modes (suitably renormalized). We isolate the contribution of evanescent modes by restricting the $\omega$-integration domain to $0 \leq \omega \leq c|\mathbf{k}|$ (so that $\kappa=\sqrt{|\mathbf{k}|^{2}-\omega^{2} / c^{2}}$ is real as it should for evanescent waves). As discussed in Sec II, zeros and poles of $D_{\mu}[\omega, \mathbf{k}]$ occur for the plasma model only for TM-polarized evanescent waves. A simple calculation leads to

$$
\begin{aligned}
& 1-\left(r_{\mathbf{k}}^{T M}\right)^{2} e^{-2 \kappa L}=\left(1-\left(r_{\mathbf{k}}^{T E}\right)^{2} e^{-2 \kappa L}\right) \times \\
& \left(\frac{g_{+}\left(\kappa^{2} L^{2}\right)-(\omega L / c)^{2}}{g_{0}\left(\kappa^{2} L^{2}\right)-(\omega L / c)^{2}}\right)\left(\frac{g_{-}\left(\kappa^{2} L^{2}\right)-(\omega L / c)^{2}}{g_{0}\left(\kappa^{2} L^{2}\right)-(\omega L / c)^{2}}\right)
\end{aligned}
$$

where the functions $g_{ \pm}(z)$ defined in $\mathrm{A} 3 \mathrm{~b}$, A3c appear. The factor involving $r_{\mathbf{k}}^{T E}$ shows no singularities for evanescent waves. With the change of variable $k \mapsto z=(\kappa L)^{2}$, we see that the two factors in the second line of (34) have simple zeros (poles) at the mode frequencies $\omega_{ \pm}\left(\omega_{0}\right)$, respectively [see Eq. $[$ A3a] ]. A calculation using the argument principle and the symmetry property $\epsilon(-\omega)=\epsilon(\omega)$ for lossless response functions, then leads straightforwardly to Eq. 32.

\section{CONCLUSION AND DISCUSSION}

In this paper we evaluate the contribution of plasmonic modes to the Casimir force using the plasma model to describe the optical response of the medium. Simple analytical expressions are found, in particular for the small and large distance asymptotics. We introduced a correction factor $\eta_{\mathrm{pl}}(L)$ that gives the plasmonic contribution to the Casimir energy, $E_{\mathrm{pl}}(L)$, in units of the Casimir energy $E_{\mathrm{Cas}}(L) \propto$ $-1 / L^{3}$ [Eq. [1]]. It turns out that $\eta_{\mathrm{pl}}(L)$ is small but positive at short distance, correctly reproducing van Kampen's result [27]. Quite surprisingly, the plasmonic contribution changes sign at the fairly short distance $L / \lambda_{\mathrm{p}} \sim 0.08$. For larger cavity lengths, $\eta_{\mathrm{pl}}(L)$ becomes negative and leads to the unusual scaling $E_{\mathrm{pl}} \propto+L^{-5 / 2}$ as $L \rightarrow \infty$. This behaviour clearly shows that the plasmonic modes are much more important for the Casimir effect than usually anticipated. They do not only dominate in the short distances limit, but they also give a large repulsive contribution at large distances.

We have calculated as well (see also [40]) the photonic mode contribution that turns out to be a monotonous function of the distance $L$ (Fig 8); it actually approaches a constant as $L \rightarrow 0$. Its large distance behaviour contains as leading order a negative $L^{-5 / 2}$ term that exactly cancels the plasmonic contribution. The Casimir energy is thus the balance of two contributions of equal magnitude which nearly cancel each other.

It would be interesting to investigate if a change in the fieldmirror coupling could somehow influence this detailed balance and therefore the value or even the sign of the Casimir force. This could be the case for nanostructured surfaces, since the plasmonic modes are associated with the electron charge density oscillations at the vacuum/metal interface. This route has already been explored within a different context, that of metamaterials in the visible frequency range. It has been shown that arrays of metallic dots or rods [42, 43, 44] exhibit a strong magnetic response in the visible, including a band with negative magnetic permeability. This behaviour arises again from plasmon modes: they are here concentrated on the metallic particles and their characteristics can be tuned with the particle shape. In the array, the plasmons delocalize and lead to a resonant electric and magnetic response. A significant modification of the Casimir force, even a change in sign, could be realistic with these materials [45].

The limits of validity of our results are imposed by the applicability of the plasma model. The approach is not intended to make quantitative predictions because the response of intra- 


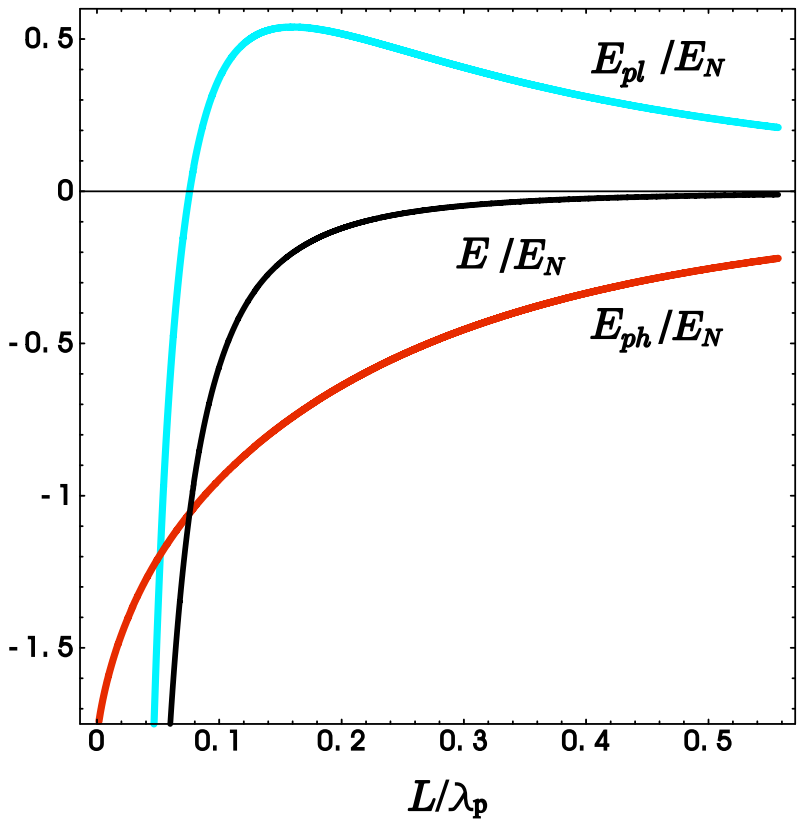

FIG. 8: A plot of plasmonic $E_{\mathrm{pl}}$, photonic $E_{\mathrm{ph}}$ and total Casimir $E$ energy vs. distance $L$, normalized to the plasma wavelength $\lambda_{\mathrm{p}}$. We normalize the energy to $(2 \pi)^{3} \frac{\hbar c \pi^{2} A}{720 \lambda_{\mathrm{p}}^{3}}$. The plasmonic energy shows a maximum for $L \approx 0.16 \lambda_{\mathrm{p}}$ (the corresponding force changes sign), while the photonic energy provides an attractive contribution at all distances.

band transitions in real metals would require a more complicated dielectric function. We are also restricted to fairly short distances where the relevant mode frequencies are sufficiently large compared to the dissipation rate. From Fig 8 , one can see, however, that the most striking effects due to plasmonic modes (change in sign and near cancellation of plasmonic and photonic contributions) indeed appear at short distances, $L \leq \lambda_{\mathrm{p}}$, where the lossless plasma response is a suitable approximation. We therefore believe that at least for this range of distances our results are quite generally valid.

In our description, the main responsible for a repulsive Casimir interaction is the plasmonic mode $\omega_{+}$. This mode crosses the border between the evanescent sector and the propagative sector, and we have it considered as being completely part of the 'plasmonic' set of modes [33]. This is an 'adiabatic' definition that is strongly suggested by the continuous change in the mode function plotted in Fig 4 . Other splittings into evanescent and photonic modes have been applied in the literature [40, 46], and we have given a brief review in Section IIIE The total Casimir energy is of course immune to these wordings. However, if one considers a structured surface, the mode branch $\omega_{+}$will change as a whole, and by analyzing this change, one could easily predict the corresponding modification of the Casimir energy.

\section{Acknowledgments}

F. I. is grateful to M. Sokolsky for providing a critical reading of the paper. F.I. enjoyed financial support from Fondazione Angelo della Riccia, from QUDEDIS, a scientific program of the European Science Foundation (ESF), and from FASTNet, a Research Training Network within the 5th Programme Framework of the European Union. F.I. and A.L. thank S. Reynaud for useful discussions.

\section{APPENDIX A: PLASMONIC DISPERSION RELATIONS}

We collect in this appendix some properties of the solutions $\omega_{ \pm}(\mathbf{k})$ of the dispersion relation Eq. (4) in the evanescent sector. Similar discussions can be found in Refs. [38, 39].

To simplify the notation, we use the dimensionless variables

$$
K=|\mathbf{k}| L, \quad \Omega=\omega L / c,
$$

and write the ratio $L / \lambda_{\mathrm{p}}=\Omega_{\mathrm{p}} / 2 \pi$. By symmetry, the mode frequencies do not depend on the angle of $\mathbf{k}$ within the mirror plane. The zeros of Eq.47) and can be split into 'odd' and 'even' cases

$$
\left\{\begin{array}{l}
1-r_{K}^{\mu}[\Omega] e^{-\kappa L}=0 \\
1+r_{K}^{\mu}[\Omega] e^{-\kappa L}=0
\end{array}\right.
$$

For the TM-polarization, the solutions to these equations can be written in the parametrized form

$$
\left\{\begin{array}{l}
K_{ \pm}=\sqrt{z+g_{ \pm}(z)} \\
\Omega_{ \pm}=\sqrt{g_{ \pm}(z)}
\end{array}\right.
$$

where

$$
\begin{aligned}
& g_{+}(z)=\frac{\Omega_{p}^{2} \sqrt{z}}{\sqrt{z}+\sqrt{z+\Omega_{\mathrm{p}}^{2}} \tanh \left(\frac{1}{2} \sqrt{z}\right)} \\
& g_{-}(z)=\frac{\Omega_{p}^{2} \sqrt{z}}{\sqrt{z}+\sqrt{z+\Omega_{\mathrm{p}}^{2}} \operatorname{coth}\left(\frac{1}{2} \sqrt{z}\right)}
\end{aligned}
$$

The parameter $z=(\kappa L)^{2}$ runs over a part of the real axis such that $z+g_{ \pm}(z) \geq 0$ in Eq. A3a): it is positive in the evanescent sector and negative in the propagating one. The square root is chosen with $\operatorname{Im} \sqrt{z}<0$. For completeness, we give here the form of $g_{+}(z)$ with negative argument:

$$
g_{+}\left(-z^{\prime}\right)=\frac{\Omega_{p}^{2} \sqrt{z^{\prime}}}{\sqrt{z^{\prime}}+\sqrt{\Omega_{\mathrm{p}}^{2}-z^{\prime}} \tan \left(\frac{1}{2} \sqrt{z^{\prime}}\right)} ;
$$

it takes positive values as long as $z^{\prime}$ is not too large (see Eqs. (A8/A9) below).

We shall also need the surface plasmon dispersion for a single metal-vacuum interface, $\Omega_{0}(K)$. It can formally be found 
by taking the limit $L \rightarrow \infty$ (while $\kappa>0$ ) in Eqs. A3b|A3c], leading to the function

$$
g_{0}(z)=\frac{\Omega_{p}^{2} \sqrt{z}}{\sqrt{z}+\sqrt{z+\Omega_{\mathrm{p}}^{2}}}
$$

that parametrizes according to the scheme of Eqs. A3a the poles of $r_{K}^{T M}[\Omega]$. We note for completeness the explicit expression for the single interface

$$
\Omega_{0}(K)=\sqrt{\frac{\Omega_{p}^{2}+2 K^{2}-\sqrt{\Omega_{p}^{4}+4 K^{4}}}{2}}
$$

(This is of course independent of $L$ since the scale factor $1 / L$ can be removed throughout.)

In the evanescent sector, both functions $z+g_{ \pm}(z)$ are monotonous so that exactly one evanescent solution is found for a given $K$. The limit $z \rightarrow \infty$ corresponds to large wave vectors $K$ where we have

$$
\Omega_{a}(K) \stackrel{K \rightarrow \infty}{\longrightarrow} \Omega_{\mathrm{p}} / \sqrt{2}, \quad a= \pm, 0 .
$$

The two surface plasmons decouple in this limit. A more detailed analysis of the asymptotic behaviour is given in Sec IIIA

The modes $\Omega_{-}(K)$ and $\Omega_{0}(K)$ remain in the evanescent sector for $K \rightarrow 0$ : analyzing the limit $z \rightarrow 0$, one gets the linear dispersions

$$
\begin{aligned}
\Omega_{-}(K) & \approx \frac{K}{\sqrt{1+2 / \Omega_{\mathrm{p}}}} \\
\Omega_{0}(K) & \approx K
\end{aligned}
$$

The mode $\Omega_{+}(K)$ crosses the light line when $z=0$, corresponding to the frequency $\Omega_{+0}=\left[g_{+}(0)\right]^{1 / 2}=$ $\Omega_{\mathrm{p}} / \sqrt{1+\Omega_{\mathrm{p}} / 2}$. The dispersion relation enters the cavity sector in a continuous and differentiable way. The same is true for the associated mode function, as illustrated in Fig 4 (inset). It reaches $K=0$ at a parameter value $z=-z_{+}$that is the solution of $z+g_{+}(z)=0$. This can be simplified to the implicit equation

$$
\sqrt{z_{+}}=\Omega_{\mathrm{p}} \cos \left(\frac{1}{2} \sqrt{z_{+}}\right)
$$

from which one gets the exact inequalities $0 \leq z_{+} \leq$ $\min \left(\Omega_{\mathrm{p}}^{2}, \pi^{2}\right)$ and the asymptotics

$$
z_{+} \rightarrow \begin{cases}\Omega_{\mathrm{p}}^{2} & \text { for } \Omega_{\mathrm{p}} \ll \pi \\ \pi^{2} & \text { for } \Omega_{\mathrm{p}} \gg \pi\end{cases}
$$

In the first case, the mode ends at the bulk mode continuum (Fig 4 is close to this situation). The second case implies that at large distance $\left(L \gg \lambda_{\mathrm{p}}\right)$, the mode stays close to the light line, as in Fig2. One can also calculate that the generic behaviour of the dispersion relation for small $K$ is quadratic:

$$
\Omega_{+}(K) \approx \Omega_{+}(0)+\frac{K^{2}}{2 \Omega_{+}(0)} \frac{\sqrt{\Omega_{\mathrm{p}}^{2}-z_{+}}-2}{\sqrt{\Omega_{\mathrm{p}}^{2}-z_{+}}+2} .
$$

[1] H. Casimir, Proc. Kon. Ned. Akad. Wet. 51, 793 (1948).

[2] M. Sparnaay, Physics in the Making, Elsevier Science Publishers, 1989.

[3] P. W. Milonni, The Quantum Vacuum, Academic Press Inc., San Diego, 1994.

[4] S. Lamoreaux, Am. J. Phys. 67, 850 (1999).

[5] M. Bordag, U. Mohideen, and V. Mostepanenko, Phys. Rep. 353, 1 (2001).

[6] K. A. Milton, J. Phys. A 37, R209 (2004).

[7] E. Lifshitz, Soviet Phys. JETP 2, 73 (1956) [J. Exper. Theoret. Phys. USSR 29, 94 (1955)].

[8] J. Heinrichs, Phys. Rev. B 11, 3625 (1975).

[9] J. Schwinger, L.L. de Raad and K.A. Milton, Ann. Phys. (N.Y.) 115, 1 (1978).

[10] S.K. Lamoreaux, Phys. Rev. A 59, R3149 (1999).

[11] A. Lambrecht and S. Reynaud, Phys. Rev. Lett. 84, 5672 (2000).

[12] A. Lambrecht and S. Reynaud, Eur. Phys. J. D 8, 309 (2000).

[13] C. Genet, A. Lambrecht and S. Reynaud, Phys. Rev. A 62, 012110 (2000).

[14] G.L. Klimchitskaya, U. Mohideen and V.M. Mostepanenko, Phys. Rev. A 61, 062107 (2000).
[15] V.B. Bezerra, G.L. Klimchitskaya and V.M. Mostepanenko, Phys. Rev. A 62, 014102 (2000).

[16] C. Genet, A. Lambrecht, and S. Reynaud, Int. J. Mod. Phys. A 17, 761 (2002).

[17] I. Pirozhenko, A. Lambrecht, and V. Svetovoy, New J. Phys. 8, 238 (2006).

[18] F. Chen, U. Mohideen, G.L. Klimchitskaya, and V.M. Mostepanenko, Phys. Rev. A 72, 020101(R) (2006).

[19] S. Raimes, Rep. Prog. Phys. 20, 1 (1957).

[20] H. Raether, Surface Plasmons on Smooth and Rough Surfaces and on Gratings, Springer, Berlin, Heidelberg, 1988.

[21] T. Ebbesen, H. Lezec, H. Ghaemi, T. Thio, and P. Wolff, Nature 391, 667 (1998).

[22] L. Martin-Moreno, F. J. Garcia-Vidal, H. J. Lezec, K. M. Pellerin, T. Thio, J. B. Pendry, and T. W. Ebbesen, Phys. Rev. Lett. 86, 1114 (2001).

[23] E. Altewischer, M. van Exter, and J. Woerdman, Nature 418, 304 (2002).

[24] A. W. C. Lau, D. Levine, and P. Pincus, Phys. Rev. Lett. 84, 4116 (2000).

[25] J. M. Obrecht, R. J. Wild, M. Antezza, L. P. Pitaevskii, S. Stringari, and E. A. Cornell, Phys. Rev. Lett. 98, 063201 (2007). 
[26] M. Antezza, L. P. Pitaevskii, S. Stringari, and V. B. Svetovoy, Phys. Rev. Lett. 97, 223203 (2006).

[27] N. V. Kampen, B. Nijboer, and K. Schram, Phys. Lett. A 26, 307 (1968).

[28] C. Genet, F. Intravaia, A. Lambrecht, and S. Reynaud, Ann. Fond. L. de Broglie 29, 311 (2004); quant-ph/0302072

[29] J. Heinrichs, Phys. Rev. B 11, 3625 (1975); erratum: Phys. Rev. B 12, 6006 (1975).

[30] P. Summerside and J. Mahanty, Phys. Rev. B 19, 2944 (1979).

[31] K. Schram, Phys. Lett. A 43, 282 (1973).

[32] E. Gerlach, Phys. Rev. B 4, 393 (1971).

[33] F. Intravaia and A. Lambrecht, Phys. Rev. Lett. 94, 110404 (2005).

[34] C. Henkel, K. Joulain, J.-P. Mulet, and J.-J. Greffet, Phys. Rev. A 69, 023808 (2004).

[35] C. Genet, A. Lambrecht, and S. Reynaud, Phys. Rev. A 67, 043811 (2003).

[36] M. Born and E. Wolf, Principles of Optics, 7th edition, Cambridge University Press, Cambridge, 1999.
[37] A. I. Markusevic, Elements of Theory of Analytic Functions, Mir, Moscow, 1988.

[38] E. N. Economou, Phys. Rev. 182, 539 (1969).

[39] D. B. Chang, R. L. Cooper, J. E. Drummond, and A. C. Young, J. Chem. Phys. 59, 1232 (1973)

[40] M. Bordag, J. Phys. A 39, 6173 (2006).

[41] A. Lambrecht, M. T. Jaekel, and S. Reynaud, Phys. Lett. A 225, 188 (1997).

[42] L. V. Panina, A. N. Grigorenko, and D. P. Makhnovskiy, Phys. Rev. B 66, 155411 (2002).

[43] V. A. Podolskiy, A. K. Sarychev, and V. M. Shalaev, J. Nonlin. Opt. Phys. Mat. 11, 65 (2002).

[44] A. N. Grigorenko, A. K. Geim, H. F. Gleeson, Y. Zhang, A. A. Firsov, I. Y. Khrushchev, and J. Petrovic, Nature 438, 335 (2005).

[45] C. Henkel and K. Joulain, Europhys. Lett. 72, 929 (2005).

[46] Z. Lenac, Phys. Rev. Lett. 96, 218901 (2006); reply: F. Intravaia and A. Lambrecht, ibid. 96, 218902 (2006). 